\title{
Sexual intimacy in later life: from the perspectives of older couples in Malaysia
}

\begin{abstract}
Aim: To identify the determinants of sexual intimacy among married older people in Malaysia. Methods: A cross-sectional study was carried out among 1294 married Malaysian older couples who were randomly selected from all 14 states in Malaysia. The data were collected by trained enumerators using a set of validated questionnaires consisting of eight sections, namely sociodemographic characteristics, chronic diseases, perceived health status, life satisfaction, body mass index, disability status (World Health Organization Disability Assessment Schedule), social support (Lubben Social Network Scale) and sexual intimacy. Results: Having good social support (AOR 0.57, 95\% CI 0.45-0.74) from family and friends were protective determinants against poor sexual intimacy in later life. Meanwhile, those who were aged 70-79 years (AOR 1.81, 95\% CI 1.35-2.42), aged >80 years (AOR 35.49, 95\% CI4.80-262.18), women (AOR 1.47, 95\% CI 1.13-1.90), non-Malay (AOR 1.93, 95\% CI1.50-2.48), received only informal education (AOR 1.81, 95\% CI 1.35-2.42), had gastritis(AOR 2.62, 95\% CI 1.58-4.34), had a stroke (AOR 3.83, 95\% CI 1.04-14.12), perceived their current health status was satisfactory (AOR 1.52, 95\% CI 1.15-2.00) and disabled based on the World Health Organization Disability Assessment Schedule (AOR 3.14, 95\% CI1.34-7.36) were at risk of poor sexual intimacy. Conclusions: The majority of older Malaysian couples were having poor sexual intimacy despite being still married and sleeping with their partners, reflecting the presence of underlying barriers towards sexual intimacy in later life among older Malaysians.
\end{abstract}

Keyword: Couple; Elderly; Malaysia; Sexual intimacy 